\title{
Uso da inteligência artificial como armamento no diagnóstico e condução clínica da estenose de canal lombar
}

\section{Use of Artificial Intelligence in Diagnosis and Clinical Conduct of Lumbar Spinal Stenosis}

\author{
Marcelo Oppermann ${ }^{1}$ Lourdes Mattos Brasil ${ }^{2}$ \\ ${ }^{1}$ Neurocirurgião pela Escola Paulista de Medicina; responsável técnico \\ do Instituto de Neurocirurgia Medullaris, Brasília; mestrando em \\ engenharia biomédica na Universidade de Brasília, Brasília, DF, Brasil \\ ${ }^{2}$ Doutora em engenharia elétrica/sistemas de informação com ênfase \\ em engenharia biomédica pela Universidade Federal de Santa \\ Catarina e coordenadora da Pós-Graduação de Engenharia \\ Biomédica da Universidade de Brasília, Brasília, DF, Brasil
}

\begin{abstract}
Address for correspondence Marcelo Oppermann, MD, Neurocirurgião, Escola Paulista de Medicina Instituto Medullaris, Brasília, DF 70910-900, Brazil (e-mail: MarceloOppermann@hotmail.com).
\end{abstract}

Arq Bras Neurocir 2016;35:18-30.

\section{Resumo \\ Palavras-Chave \\ - estenoses de canal lombar \\ - inteligência artificial \\ - sistema especialista \\ - diagnóstico \\ - tratamento}

A estenose do canal vertebral lombar (ECL) é uma patologia complexa, com alta incidência entre pessoas acima de 65 anos de idade. No entanto, o diagnóstico correto é, por vezes, difícil de ser confirmado. O uso de modelos de Inteligência Artificial (IA) na medicina é, em geral, desconhecida para a maioria da comunidade médica, mas tem sido usada há décadas na assistência em UTI, os métodos de imagem e dispositivos de diagnóstico eletrônico (ECG). Através de uma revisão sistemática da literatura, com foco nos achados clínicos e radiológicos, juntamente com todas as modalidades de tratamento, foi possível identificar o ambiente completo de pacientes LSS, para responder a quatro questões: (a) "Com base no quadro clínico, o paciente tem um, cenário moderado ou grave?”; (b) "Com base nos dados radiológicos, o paciente pode ser classificado com um cenário leve, moderada ou grave?”; (c) “Qual é a probabilidade, com base na anamnese, do paciente ter ECL?”; (d) "Qual é o melhor tratamento a ser oferecido?”.+Com o auxílio de um software usando Sistema Especialista (Expert Sinta), uma linguagem de IA, alocamos todas as variáveis e seus valores para orientar o software responder às quatro perguntas. Foi possível identificar 657 artigos científicos, no entanto apenas 63 poderia mencionar não apenas as variáveis, mas a sua probabilidade de ocorrência ou teve disponibilidade texto completo. Foi possível classificar a intensidade do quadro clínico e radiológico, criar um índice de probabilidade para LSS e oferecer o melhor tratamento. Recomendamos o uso, sob supervisão received

July 6,2015

accepted

October 21, 2015

published online

December 28, 2015
DOI http://dx.doi.org/

$10.1055 / \mathrm{s}-0035-1570364$. ISSN $0103-5355$.
Copyright $(2016$ by Thieme Publicações License terms Ltda, Rio de Janeiro, Brazil
(1) $\Theta \circledast$ 
médica, em de Neurocirurgia ou clínicas ortopédicas como um conselheiro para os pacientes com ELA.

Abstract

The lumbar spinal stenosis (LSS) is a complex pathology with high incidence among people above 65 years old. However, the correct diagnose is sometimes difficult to perform. The use of Artificial Intelligence (Al) models in medicine is, in general, unfamiliar for the majority of medical community, but has been used for decades in assistance in ICUs, image methods and electronic diagnostic devices (EKG). Through a systematic literature review focused in the clinical and radiological findings, in addition to all treatment modalities, we identified the complete environment of LSS patients, to answer four questions. (a) "Based on the clinical presentation, the patient has a mild, moderate or severe scenario?", (b) "Based on the radiological data, the patient can be classified having a mild, moderate or severe scenario?", (c) "What is the probability, based on the anamneses, the patient has LSS?", and (d) "What is the best treatment to be offered?". With the aid of a software using Expert System (Expert Sinta), a language of $\mathrm{Al}$, we allocate all the variables and their values to orient the software to answer the

\section{Keywords}

- lumbar spinal stenosis

- artificial intelligence

- expert system

- diagnosis

- treatment four questions. It was possible to identify 657 scientific articles, however only 63 could mention not only the variables, but their occurrence probability or had full text availability. It was possible to classify the intensity the clinical and radiological scenario, create a probability index for LSS and offer the best treatment. We recommend the use, under medical supervision, in neurosurgery or orthopedic clinics as an adviser for patients with LSS.

\section{Introdução}

É de consenso público que a população mundial está envelhecendo. Estatísticas americanas apontam que atualmente $11 \%$ da população estão acima de 60 anos de idade e no ano de 2050 este número elevar-se-á para $22 \%{ }^{1}$ O fato de termos melhor expectativa de vida cria uma íntima relação com o melhor nível educacional, que por sua vez, traz maior acesso a novas tecnologias, entre elas o serviço médico. Infelizmente, o fato de nos tornarmos mais idosos leva-nos a um estado, inevitável e indesejável, de doença. Inúmeras patologias estão associadas com o envelhecimento do ser humano. Câncer, acidente vascular cerebral, dentre outras, apresentam ligação direta com a faixa etária da terceira idade. Assim é a estenose de canal lombar (ECL), patologia que afeta o canal vertebral da coluna lombar do idoso.

A coluna vertebral, no ser humano, apresenta propriedades e estruturas que em conjunto definem suas três principais funções. Primeiro, estabilidade, devido a um conjunto de ossos, ligamentos e músculos arranjados, que conseguem manter uma pessoa em posição ereta com segurança. Segundo, flexibilidade, que através de articulações e ligamentos permite a adoção de inúmeras posições sem risco à estabilidade. Por último, proteção: através de um canal ósseo responsável por manter a medula e suas raízes com segurança em seu interior contra agentes nocivos externos.

A estenose do canal lombar refere-se a um estreitamento de fora para dentro do canal que mantém todo o tecido neural da coluna. 0 termo estenose é derivado do grego antigo, e é mais bem traduzido como "estreito". Verbiest declarou, referindo-se ao canal vertebral, como sendo um "estreitamento anormal de uma cavidade, sendo capaz de produzir doença em seus constituintes internos." ${ }^{2}$

Os sintomas clínicos, de dores lombares com ou sem irradiação para os membros inferiores, juntamente com claudicação neurogênica, levam à suspeição clínica da doença. Estes, acrescidos aos exames de imagem, ajudam a defini-la de forma aproximada. Entretanto, os sintomas referidos para esta patologia são muito inespecíficos, já que inúmeras causas podem ser responsáveis pela dor lombar e/ou dor irradiada para os membros. Doze por cento da população jovem e $21 \%$ dos representantes da terceira idade apresentam este conjunto de sintomas, mas nem todos têm $\mathrm{ECL}^{3}$ Doenças do canal vertebral, como hérnias, doenças musculoesqueléticas como sacroileíte, bursite de quadril, e até outras síndromes reumatológicas, assemelham-se à estenose vertebral. Em resumo, não há, até o momento, uma ferramenta como Gold-Standard que valide e defina de forma acertada esta patologia. ${ }^{4}$

A inteligência artificial (IA) tem sido utilizada em sistemas matemáticos complexos há muito tempo, e ultimamente, na medicina. Modelos inteligentes têm sido aplicados com sucesso em diversas áreas da medicina, como sistemas de diagnóstico, análise biomédica, análise de imagens e descoberta de novas drogas, bem como para resolver os desafios e as políticas de desenvolvimento de drogas. ${ }^{5-7}$ Recentemente, a neurocirurgia aderiu a esta ferramenta como diagnóstico e no auxílio para condução terapêutica. ${ }^{8-11}$

O objetivo deste trabalho é fazer uma revisão dos melhores indicadores (sinais e sintomas) para o correto diagnóstico da estenose de canal lombar, e através da utilização de uma ferramenta da inteligência artificial, o Sistema Especialista, proporcionar ao entrevistador (médico ou 
profissional da saúde) dados como: a probabilidade do paciente entrevistado ter a doença e a melhor forma de conduzir seu caso.

\section{Materiais e Métodos}

\section{Dados}

Através de uma pesquisa na base de dados MEDLINE (ferramenta de busca PubMed), procurou-se por artigos de revisão e/ou originais que fizessem referência aos sintomas e sinais mais prevalentes em pacientes portadores da ECL, bem como por indicadores radiológicos e a condução clínico/cirúrgica para cada situação. As referências foram escolhidas desde que tivessem dados como: probabilidade de sua incidência na patologia e/ou importância no diagnóstico ou tratamento.

Não foi feita qualquer distinção do valor estatístico do trabalho (medicina baseada em evidência), a fim de se evitar desqualificar ou desprezar o real valor de artigos que somente relatem seus casos. Tais artigos para muitos apresentam grande valia, pois remetem a um dado que pouco pode ser mensurado, a experiência clínica e o relato dos pesquisadores. $^{12,13}$

Procurou-se por indicadores, na história clínica, mais prevalentes em pacientes sabidamente portadores desta doença. Assim, pode-se traçar uma incidência para cada dado adquirido e avaliar seu peso para o correto diagnóstico. Neste quesito, buscou-se por Lumbar Spinal Stenosis e uma destas palavras: Clinical Scenario ou Symptoms ou Diagnostic ou Diagnose. Os dados radiológicos mais prevalentes nos raios $\mathrm{X}$, tomografia e ressonância foram agrupados e separados por sua importância clínica e estatística no paciente. Neste aspecto, procurou-se por Lumbar Spinal Stenosis e uma destas palavras: $x$-Ray ou MRI ou Tomography ou Radiologic Diagnose.

Pesquisou-se, ainda, os tipos de tratamento, e quais eram suas principais indicações, frente a cenários bem característicos nos artigos clínicos e de revisões. Foram incluídas as modalidades clínicas, desde medicação até fisioterapia, bem como os procedimentos cirúrgicos. Neste aspecto, procurouse por Lumbar Spinal Stenosis e uma destas palavras: Treatment ou Consevative ou Surgery ou Minimally Invasive ou Physioterapy ou Medication.

\section{Sistema Especialista}

Para a construção do protocolo em sistema especialista, utilizou-se o software Expert Sinta v1.1b (Grupo Sinta, Fortaleza, Brasil - Freeware). O software consegue criar um direcionamento para o entrevistador (médico), onde através de questões diretas fornece uma ideia do estado constitucional da doença do entrevistado (paciente), junto com o tratamento recomendado. Para tanto, requer a criação de variáveis e seus valores. Um exemplo de variável seria a escala visual de dor, VAS (Visual Analitic Scale), que é uma forma de quantificar em números o grau de dor sofrido pelo doente. Os valores para esta variável seriam números de zero a dez, e assim por diante. Após a criação destas variáveis, torna-se imprescindível a estipulação de regras para que estas variáveis e seus valores possam ser úteis, fornecendo assim já um diagnóstico ou um parecer sobre algum dado. No final, com as regras criadas, pode-se estipular um objetivo ou, mais especificamente, conseguir que o sistema especialista avalie, nesta situação, a probabilidade do diagnóstico, sua gravidade, e proponha o tratamento.

Separaram-se, então, os dados de acordo com sua importância, nas seguintes categorias: sintomas, sinais, doenças associadas, sinais radiológicos. A separação dos dados foi importante para alimentar o software escolhido, e assim responder a quatro perguntas essenciais:

1. Frente ao sintomas e sinais apresentados pelo paciente, ele é portador de uma doença leve, moderada ou grave?

2. Frente aos dados radiológicos encontrados, o paciente é portador de uma doença leve, moderada ou grave?

3. Frente a todos os indicadores clínicos e radiológicos, a probabilidade do paciente ter estenose de canal lombar é improvável, baixa, moderada ou alta?

4. Frente ao cenário apresentado, qual é a melhor terapêutica para este paciente?

\section{Resultados}

\section{Dados da Literatura}

A pesquisa encontrou 657 artigos (254 sobre diagnóstico e 403 sobre tratamento) que faziam referência de alguma forma aos termos utilizados. Entretanto, ateve-se a 86 (distribuídos conforme a - Tabela 1) que faziam menção à incidência e possibilitavam acesso completo.

Analisando os artigos, foi possível separar os cenários clínicos que mais pudessem representar a patologia da estenose de canal vertebral, e paralelamente, os que pudessem excluí-la. Foram identificados: idade, presença de dor lombar, radiculopatia, claudicação neurogênica, dor em nádegas e em membros inferiores bilateralmente, ausência de dor quando sentado, distúrbio urinário de causa não genitourinária, melhora da dor quando tronco flexionado e presença de sintomas de cauda equina.

Do ponto de vista radiológico, conseguiu-se identificar nas modalidades de raios $\mathrm{X}$, tomografia e ressonância

Tabela 1 Tipo e quantidade de artigos nos estudos encontrados

\begin{tabular}{|l|l|}
\hline \multicolumn{2}{|l|}{ Estudos encontrados } \\
\hline Tipo & Quantidade \\
\hline Revisão & 22 \\
\hline Observacional retrospectivo & 27 \\
\hline Observacional prospectivo & 19 \\
\hline Randomizado & 11 \\
\hline Caso controle & 3 \\
\hline Análise multivariada & 1 \\
\hline Estudo de caso & 1 \\
\hline Comentário & 2 \\
\hline Total & 86 \\
\hline
\end{tabular}


magnética os seguintes achados: variações de estenose foraminal, de recesso lateral e de canal vertebral, bem como o sinal de sedimentação radicular. Como diagnóstico diferencial, fez-se menção a presença de diabetes e de insuficiência vascular. No quesito condução e tratamento, encontrou-se referência ao tratamento fisioterápico, infiltração peridural e cirurgia. E dentro do campo cirurgia foram reportadas as modalidades: dispositivo interespinhoso, laminectomia, laminoplastia, cirurgia minimamente invasiva e laminectomia com artrodese. A - Tabela 2 lista as variáveis junto aos possíveis valores encontrados.

\section{Sistema Especialista}

Cada variável, com suas respostas (-Tabela 2 ), poderia ser univalorada, se o valor escolhido já excluísse o outro da mesma variável; ou multivalorada, caso permitisse mais de um valor foi criada, perfazendo 29 variáveis e 85 valores. De forma prática, um paciente não pode ter um VAS $\geq 7$ e ao mesmo tempo <7; assim sendo, o VAS é univalorável. A - Fig. 1 mostra a criação de duas variáveis, sendo a primeira univalorada e a segunda multivalorada.

Os objetivos, quando criados, tinham a intensão de responder as quatro perguntas indicadas na - Fig. 2, que faz referência aos questionamentos feitos (1-4) e os apresenta junto com suas respostas, para que a pergunta sobre 0 cenário clínico seja respondido (e).

\section{Cenário Clínico}

Os sintomas da ECL restringem-se a dor lombar ou irradiada para os membros inferiores (MMII), associada ou não com disestesias, perda de força muscular e claudicação. A fim de medir a dor, foi utilizada com maior frequência a escala visual de dor VAS. ${ }^{4,14-17}$ A escala sintomática e física de Zurique foi utilizada de acordo com seus resultados na classificação da gravidade em alguns estudos. ${ }^{18-21}$ Frente às variáveis clínicas (sinais e sintomas), conseguiu-se estipular, de acordo com a nossa bibliografia pesquisada, três tipos de cenários aos acometidos. A - Tabela 3 mostra com detalhes estes quesitos.

\section{Cenário Radiológico}

Para conseguir definir os reais indicadores radiológicos, que pudessem ser importantes na confirmação da doença e quantificação da gravidade, foi decidido dividi-los por tipo de exame: raios $X$, tomografia e ressonância. Assim, foi possível utilizar o real valor de cada método na classificação radiológica para a ECL. Através dos raios X, foi identificado, de importante, a presença de listese e instabilidade dinâmica. ${ }^{22-28}$ A tomografia teve seu valor na identificação do diâmetro do recesso lateral. ${ }^{29,30} \mathrm{E}$ a ressonância, na classificação da doença foraminal ${ }^{31}$ e no sinal de sedimentação, ${ }^{32-36}$ entre outros parâmetros. ${ }^{37,38}$ A - Tabela 4 detalha as variáveis e seus valores.

\section{Probabilidade}

Para responder a terceira pergunta, foi utilizada a relevância de cada sintoma, sinal ou dado radiológico, criando-se dessa forma uma escala de pontos para a sua presença, conforme a - Tabela 5. O princípio foi estipular a probabilidade da ECL estar presente no paciente examinado. Foram 23 valores com 768 combinações possíveis que podem variar de -6 a +23 pontos. Os valores para cada variável (indicador) foram calculados através de um processo de probabilidade cuja ocorrência realmente estivesse relacionada à presença da ECL. Este cálculo foi feito utilizando o processo de lógica Fuzzy, ideal para modelamento de múltiplas variáveis (-Tabela 5). Por exemplo, o simples fato do paciente ter mais que 70 anos de idade aumenta a chance de ter ECL em $70 \%$. Já a ausência de claudicação diminui em $80 \%$ o fato. ${ }^{14,15}$ A - Tabela 6 resume o resultado.

\section{Tratamento}

Para a orientação do tratamento, as questões anteriores (-Fig. 2) tiveram que ser primeiramente respondidas. Utilizaram-se as variáveis já descritas (clínica e radiológicas) junto com as escalas criadas de graduação da doença (-Tabelas 3 e 4) e sua probabilidade de estar ou não presente (-Tabela 5). Consideraram-se as seguintes modalidades de tratamento: fisioterapia, ${ }^{39-42}$ infiltração peridural, ${ }^{43-45}$ e cirurgia. Esta contempla o dispositivo interespinhoso, ${ }^{21,46-50}$ a cirurgia minimamente invasiva, ${ }^{51-53}$ a laminectomia sem artrodese $\mathrm{e}^{22,23,54-63}$ e a laminectomia com artrodese. ${ }^{25,64-70}$ Utilizou-se ainda uma escala que avalia a resposta ao tratamento inicial instituído. ${ }^{4,17-19,21}$ Aqui a inteligência artificial mostrou seu grande valor, pois a escolha do tratamento dependeu de inúmeras variáveis, o que torna quase incompreensível a construção terapêutica por um algoritmo. A construção obedeceu às orientações presentes na - Tabela 7.

\section{Discussão}

O diagnóstico preciso e a condução terapêutica da estenose de canal lombar merecem muita discussão, prova disso é a vasta literatura sobre o tema. A inteligência artificial, na medicina, já tem seu papel bem estabelecido. Seu uso permeia inúmeros processos eletrônicos, com intuito de melhorar o diagnóstico e auxiliar na escolha terapêutica. Embora seu benefício, tem-se ainda uma parca difusão nas patologias da coluna vertebral, restrita a poucos estudos. 8,11

Neste artigo, o objeto de análise e estruturação é uma nova forma de ajudar o médico-cirurgião de coluna (neurocirurgião ou ortopedista) a conduzir da melhor maneira a entrevista médica, e a oferecer a opção terapêutica mais adequada, através de um software chamado Sistema Especialista, que utiliza a inteligência artificial como ferramenta. O software Expert Sinta conduz o especialista, através da criação de variáveis, valores e objetivos, a um tratamento protocolar padronizado, de acordo com a necessidade de cada paciente. Não foi encontrado nenhum trabalho na literatura médica utilizando essa ferramenta para a ECL.

As variáveis aqui empregadas têm como primeiro objetivo estabelecer a gravidade clínica na qual o paciente está inserido. Foram utilizados nesta caracterização a quantificação da dor lombar com a VAS, a presença de sintomas e sinais neurológicos, a presença de claudicação e 


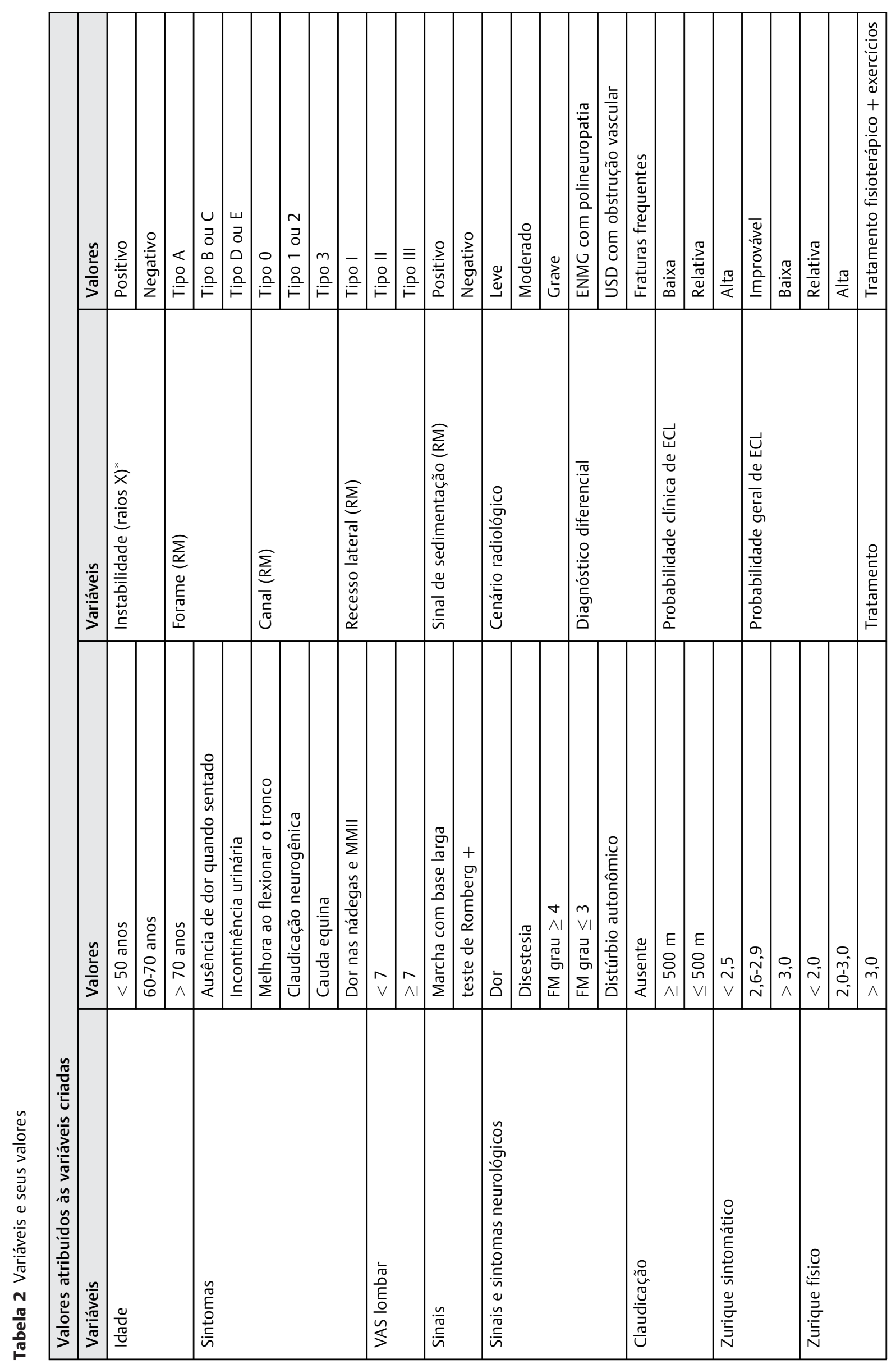




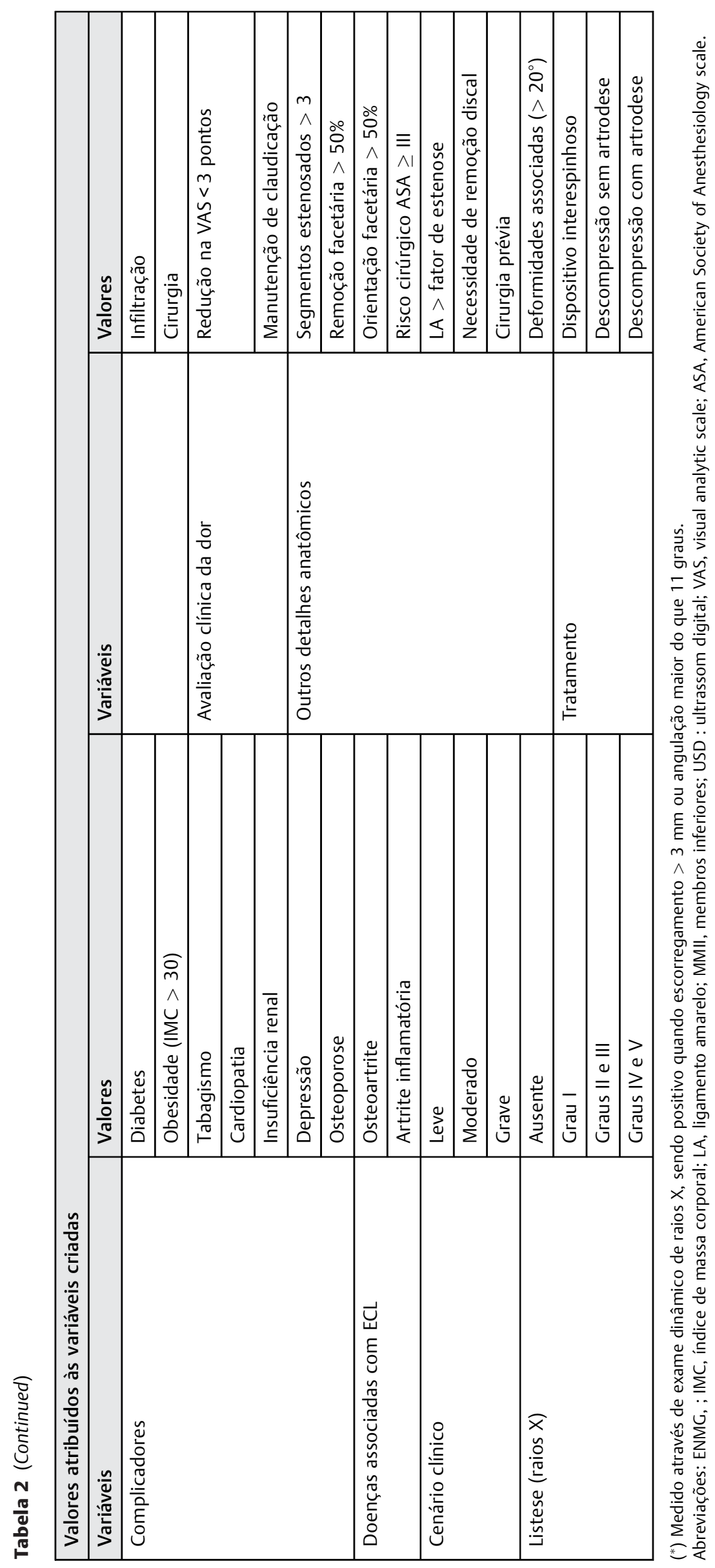


24 Inteligência artificial na estenose de canal lombar Oppermann, Brasil

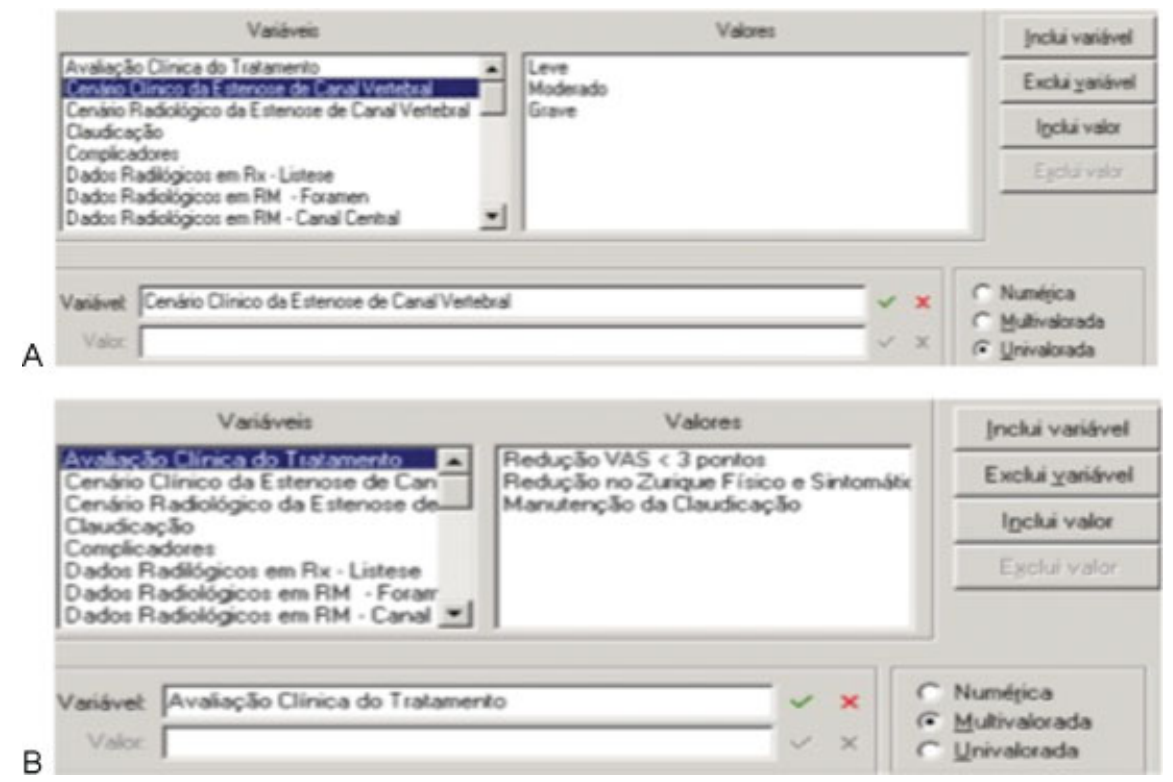

Fig. 1 Variáveis univaloradas (a) e (b) multivaloradas.
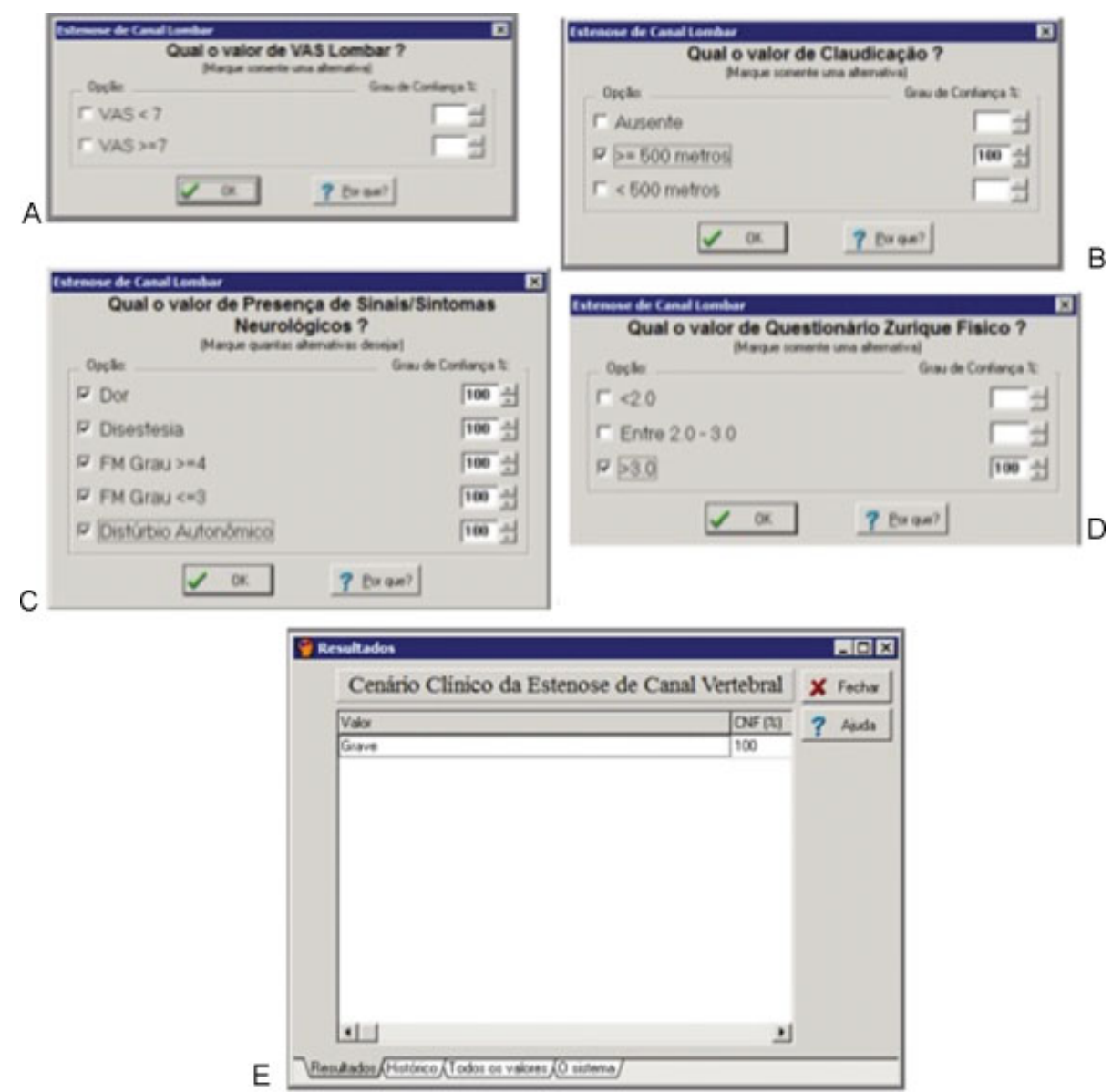

Fig. 2 Processo de perguntas oferecidas (1-4) a fim de estabelecer o cenário clínico.

sua quantificação (distância), e a escala baseada no Questionário Zurique Sintomático e Físico. ${ }^{18,19}$ Este, já validado, refere-se aos sintomas e déficits funcionais dos pacientes acometidos pela ECL e quantifica-os, sendo que a parte sintomática tem valores variando de 1,0 a 5,0 e a física de 1,0 a 4,0. Com estas variáveis e seus valores, é possível estratificar os dados da anamnese em uma das categorias de gravidade (-Tabela $\mathbf{3}$ ).

A seguir, optou-se por estabelecer a necessidade de classificar os dados radiológicos novamente, por gravidade. 
Tabela 3 Classificação do cenário clínico

\begin{tabular}{|c|c|c|c|c|}
\hline \multicolumn{5}{|l|}{ Cenário clínico da ECL } \\
\hline \multirow[t]{2}{*}{ Variáveis } & \multirow[t]{2}{*}{ Valores } & \multicolumn{3}{|c|}{ Resultado } \\
\hline & & Leve & Moderado & Grave \\
\hline \multirow[t]{2}{*}{ VAS } & $<7$ & $x$ & & \\
\hline & $\geq 7$ & & $x$ & $X$ \\
\hline \multirow[t]{2}{*}{ S \& S neurológicos* } & Ausentes & $X$ & $x$ & \\
\hline & Presentes & & & $\mathrm{X}$ \\
\hline \multirow[t]{3}{*}{ Claudicação } & Ausente & $x$ & & \\
\hline & $>500 \mathrm{~m}$ & & $x$ & \\
\hline & $<500 \mathrm{~m}$ & & & $\mathrm{X}$ \\
\hline \multirow[t]{3}{*}{ Zurique sintomático } & $\leq 2,5$ & $x$ & & \\
\hline & $2,6-3,0$ & & $X$ & \\
\hline & $>3,0$ & & & $x$ \\
\hline \multirow[t]{3}{*}{ Zurique físico } & $<2$ & $x$ & & \\
\hline & $2,0-3,0$ & & $x$ & \\
\hline & $>3,0$ & & & $X$ \\
\hline
\end{tabular}

$\left(^{*}\right)$ S\&S: Sinais e Sintomas.

Abreviações: ECL, estenose de canal lombar; VAS, visual analytic scale.

Utilizou-se o exame de raios $\mathrm{X}$ como forma de identificar a presença de listese ou instabilidade no exame dinâmico. ${ }^{23,71,72}$ Pela tomografia e ressonância magnética, conseguiu-se definir o grau de comprometimento dos recessos laterais vertebrais, ${ }^{29,30,73,74}$ quão grave é a estenose foraminal ${ }^{31,75-77}$ ou o canal vertebral. ${ }^{37,38}$ Outro método utilizado foi o chamado sinal de sedimentação das raízes. ${ }^{32-35}$ Este define como positivo ou negativo um sinal indireto causado pela estenose, ou seja, quanto menor o canal vertebral mais juntas estarão as raízes lombares. Em suma, com todos esses dados radiológicos, há possibilidade de se definir o grau de comprometimento vertebral, conforme exposto na - Tabela 4.

Houve, então, necessidade de se confirmar a presença da doença estenótica lombar no indivíduo testado. Assim, utilizaram-se inúmeros artigos que faziam menção à incidência ou à probabilidade de o paciente ter realmente a doença

Tabela 4 Classificação quanto ao cenário radiológico

\begin{tabular}{|c|c|c|c|c|}
\hline \multicolumn{5}{|c|}{ Cenário radiológico da ECL } \\
\hline \multirow[t]{2}{*}{ Variáveis } & \multirow[t]{2}{*}{ Valores } & \multicolumn{3}{|c|}{ Resultado } \\
\hline & & Leve & Moderado & Grave \\
\hline \multirow[t]{3}{*}{ Forame } & Tipo A & $x$ & & \\
\hline & Tipos B e C & & $x$ & \\
\hline & Tipos D e E & & & $x$ \\
\hline \multirow[t]{3}{*}{ Recesso lateral } & Tipo I & $X$ & & \\
\hline & Tipo II & & $x$ & \\
\hline & Tipo III & & & $x$ \\
\hline \multirow[t]{3}{*}{ Canal central } & Tipo 0 & $x$ & & \\
\hline & Tipos 1 e 2 & & $x$ & \\
\hline & Tipo 3 & & & $x$ \\
\hline \multirow[t]{2}{*}{ Sinal de sedimentação } & Positivo & $x$ & $x$ & \\
\hline & Negativo & & & $x$ \\
\hline
\end{tabular}


Tabela 5 Valoração em pontos de cada indicador na classificação de probabilidade de ECL

\begin{tabular}{|c|c|}
\hline \multicolumn{2}{|l|}{ Escala de classificação da ECL } \\
\hline Variável & Pontos \\
\hline Idade $<50$ & -2 \\
\hline Idade $50-70$ & 1 \\
\hline Idade $>70$ & 3 \\
\hline Dor lombar & 1 \\
\hline Radiculopatia & 2 \\
\hline Claudicação & 2 \\
\hline Dor nas nádegas e MMII & 2 \\
\hline Ausência de dor quando sentado & 3 \\
\hline Distúrbio urinário & 3 \\
\hline Melhora quando flexionado & 3 \\
\hline Cauda equina & 3 \\
\hline Ausência de claudicação & -5 \\
\hline Radiologia & 1 \\
\hline \multicolumn{2}{|l|}{ Foraminal tipo A } \\
\hline \multicolumn{2}{|l|}{ Recesso tipo I } \\
\hline \multicolumn{2}{|l|}{ Central tipo 0} \\
\hline \multicolumn{2}{|l|}{ Sinal de sedimentação negativo } \\
\hline Radiologia moderada & 3 \\
\hline \multicolumn{2}{|l|}{ Foraminal tipos B e C } \\
\hline \multicolumn{2}{|l|}{ Recesso tipo II } \\
\hline \multicolumn{2}{|l|}{ Central tipos 1 e 2} \\
\hline Radiologia grave & 5 \\
\hline \multicolumn{2}{|l|}{ Foraminal tipos $\mathrm{D}$ e $\mathrm{F}$} \\
\hline \multicolumn{2}{|l|}{ Recesso tipo III } \\
\hline \multicolumn{2}{|l|}{ Central tipo 3} \\
\hline Sinal de sedimentação positiva & \\
\hline
\end{tabular}

quando o sinal estivesse presente. 0 trabalho de Suri et al., ${ }^{15}$ entre outros, ${ }^{4,16,17}$ foi importante para definir o real valor de um sintoma ou sinal encontrado. Um bom exemplo é o simples fato de o paciente não apresentar claudicação, demonstrar que ele tem cerca de $2,3 \%$ de chances de ter ECL. Já a ausência de dor quando sentado é um sinal ampla-

Tabela 6 Contagem dos pontos apresentados na - Tabela 5 e sua associação à probabilidade da ECL

\begin{tabular}{|l|c|}
\hline \multicolumn{2}{|l|}{ Probabilidade de ECL } \\
\hline Resultado & Pontuação \\
\hline Improvável & $\leq 3$ \\
\hline Baixa & 4 a 6 \\
\hline Relativa & 7 a 9 \\
\hline Alta & $\geq 10$ \\
\hline
\end{tabular}

mente positivo para confirmar o diagnóstico (74\%). Através dessas probabilidades, foi possível criar uma escala variando de -6 a +23 pontos. Por um processo de análise do cruzamento de todas as variáveis (768 cenários no total), chegouse aos indicadores da probabilidade de um indivíduo ter a doença (-Tabela 6). Assim, dificilmente um paciente terá ECL se obtiver menos que 3 pontos; por outro lado, ele terá alta probabilidade se somar mais de 10 pontos.

Para definir o processo, verificou-se a conduta terapêutica. Foram considerados como opções: a fisioterapia, a medicação, a infiltração e a cirurgia. De acordo com a descrição de estudos que utilizam a fisioterapia como método terapêutico voltado para pacientes com estenose de canal lombar, ela é eficaz em pacientes com quadros mais leves e sempre estará na primeira linha, junto com a medicação. As técnicas aconselhadas englobaram a terapia manual, o método McKenzie, exercícios físicos com utilização de movimentos isométricos e a caminhada com suporte de peso. O tempo recomendado ficou em torno de 6 semanas. Kreiner et al., em sua diretriz, definiram que não há nenhum estudo mostrando valor ou não no uso de medicações para a ECL. O mesmo valeu para a fisioterapia, mas os autores aconselham seu uso para pacientes com caso de leve a moderado. $^{43}$

Outro método terapêutico utilizado neste estudo foi a infiltração peridural. ${ }^{44,45}$ Nesta técnica, foram avaliados o acesso foraminal, o interlaminar pela linha média, e o interlaminar parassagital. O primeiro exibe uma excelente distribuição medicamentosa, quando comparado a outras técnicas, mas com alto risco de intercorrências potencialmente graves (isquemia medular, embolia). ${ }^{78} \mathrm{O}$ acesso interlaminar pela linha média tem grande difusão de uso entre os especialistas, mas a distribuição medicamentosa não é adequada quanto ao acesso interlaminar parassagital. ${ }^{45}$ Assim, esta é a técnica por nós orientada no programa Expert Sinta. A recomendação é feita nos cenários leves e moderados, sempre que haja falha nas 6 primeiras semanas de tratamento fisioterápico, conforme a classificação de falha. 3,19,21 Vale ressaltar que todos os pacientes encaminhados para infiltração são seguidos pelo acompanhamento da fisioterapia, e o processo infiltrativo poderá ser repetido até 3 vezes com intervalos mínimos de 15 dias.

No final, incluímos o processo cirúrgico com sua gama de possibilidades. Como recomendação geral, a cirurgia foi proposta a pacientes do cenário clínico moderado que apresentaram falha ao tratamento conservador, e a todos os do cenário grave. O estudo SPORT confirmou que, dentro do período de 8 anos após a cirurgia, os pacientes operados obtiveram melhores resultados, em comparação aos tratados somente com fisioterapia. ${ }^{79}$ Esta assertiva corrobora outros estudos. $^{70,80-83}$ Assim, a indicação para pacientes com cenário clínico e radiológico grave é a cirurgia.

A opção pelo procedimento ideal obedeceu algumas diretivas, sendo que a presença ou ausência dos indicadores leva à escolha da melhor técnica cirúrgica, conforme mostra a - Tabela 7. De maneira geral o dispositivo interespinhoso foi recomendado a pacientes mais idosos, com risco cirúrgico desfavorável, envolvimento estenótico mais restrito ao canal 
Tabela 7 Resumo da conduta terapêutica de acordo com as variáveis clínicas e radiológicas encontradas

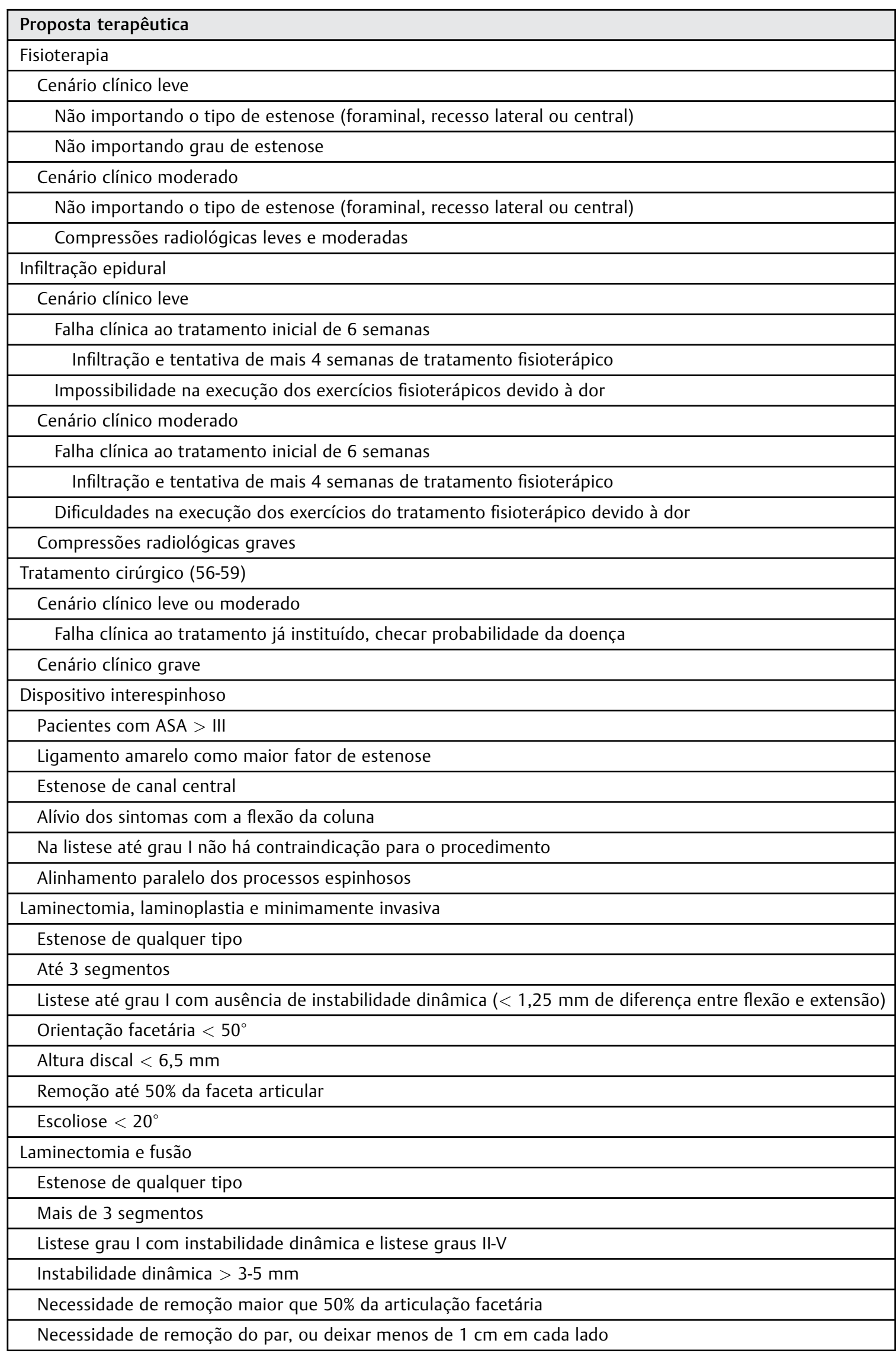


Tabela 7 (Continued)

\begin{tabular}{|l|}
\hline Proposta terapêutica \\
\hline Sinais de instabilidade intraoperatória \\
\hline Necessidade de remoção do disco intervertebral \\
\hline Osteoporose $\left(\mathrm{IMC}<0,62 \mathrm{mg} / \mathrm{cm}^{2}\right)$ \\
\hline Escoliose $>20^{\circ}$ \\
\hline Presença de grande componente mecânico na dor lombar \\
\hline Reoperação por falha radiológica ou clínica \\
\hline
\end{tabular}

vertebral e decorrente de hipertrofia de ligamento amarelo. Dentre as contraindicações para o procedimento, incluíramse presença de malformação do processo espinhoso e presença de sintoma radicular importante, sendo o forame o maior contribuinte para o mesmo. A laminotomia, laminectomia e técnica endoscópica têm sua indicação para a maior parte dos procedimentos que não apresentem sinais de instabilidade, ou conjunto de fatores que levem à instabilidade no pós-operatório. Blumenthal et al., analisando quarenta pacientes de forma retrospectiva, observaram alguns achados como indicadores de instabilidade. ${ }^{23}$ Angulo facetário $>50^{\circ}$, altura discal $>6,25 \mathrm{~mm}$ e espondilolistese dinâmica $>1,25 \mathrm{~mm}$, isoladamente, já poderiam ser responsáveis por instabilidade, mas se os três indicadores estivessem presentes a chance de reoperação com finalidade de fixação foi de $75 \%$. De maneira geral, estas técnicas que não utilizam instrumental para fixação, no nosso protocolo, foram indicadas para qualquer grau de estenose em até três níveis articulares, com ausência de sinais de instabilidade ou com potencial para tal, ${ }^{23}$ com deformidades estruturais menores que $20^{\circ}$ (por exemplo, escoliose), ou quando há possibilidade de boa descompressão deixando no mínimo $50 \%$ das facetas íntegras. A laminectomia associada à fusão com instrumentação ficou reservada para as situações remanescentes. De modo geral, seu grande benefício ocorre sempre que houver chance de o procedimento de descompressão causar instabilidade, ou alterar de forma acentuada a curvatura nos eixos coronal e sagital. Incluem: descompressão maior que três níveis, listese com grau > II, instabilidade dinâmica, remoção maciça das estruturas ósseas e necessidade de remoção do disco intervertebral. Outro fator importante para a artrodese é a existência de um grande componente de dor mecânica no quadro clínico apresentado pelo paciente, pois a simples descompressão pode até agravar a dor de base. ${ }^{82}$

Apesar de ser este o protocolo utilizado em nosso Instituto, e de ele ter sido embasado por extensa revisão bibliográfica, é fato que ainda não apresenta validação em estudo apropriado, o que recomenda cautela em sua utilização nos serviços de neurocirurgia. Outro detalhe importante é que ele não contempla respostas para algumas situações encontradas na clínica diária, como a associação a trauma, obesidade, ${ }^{84}$ fatores psiquiátricos ${ }^{85}$ e distúrbios do balanço sagital. ${ }^{86}$ Como todo sistema inteligente matemático, deve ser acompanhado e fiscalizado por profissionais qualificados.

\section{Conclusão}

Este trabalho, pelo conhecimento de sua equipe, foi o primeiro estudo que envolveu o Sistema Especialista como ferramenta no diagnóstico, classificação e condução da doença estenótica lombar. A inteligência artificial já é amplamente utilizada na medicina em aparelhos de eletrocardiograma e respiradores, entre tantos outros. ${ }^{5-7}$ Recentemente, a neurocirurgia tem-se utilizado dos benefícios desse sistema na condução de algumas doenças, sendo seus resultados promissores. ${ }^{8-11}$ Conseguiu-se, de forma eficiente e prática, mostrar que esta opção terapêutica pode auxiliar em clínicas neurocirúrgicas ou ortopédicas, orientando o médico e padronizando suas condutas, mas sempre respeitando seu feeling em cada situação.

\section{References}

1 United Nations population Fund - UNFPA. Ageing in the TwentyFirst Century. New York: UNFAP; 2012

2 Verbiest $\mathrm{H}$. Results of surgical treatment of idiopathic developmental stenosis of the lumbar vertebral canal. A review of twentyseven years' experience. J Bone Joint Surg Br 1977;59(2):181-188

3 Hicks GE, Gaines JM, Shardell M, Simonsick EM. Associations of back and leg pain with health status and functional capacity of older adults: findings from the retirement community back pain study. Arthritis Rheum 2008;59(9):1306-1313

4 Konno S, Kikuchi S, Tanaka Y, et al. A diagnostic support tool for lumbar spinal stenosis: a self-administered, self-reported history questionnaire. BMC Musculoskelet Disord 2007;8:102

5 Sheikhtaheri A, Sadoughi F, Hashemi Dehaghi Z. Developing and using expert systems and neural networks in medicine: a review on benefits and challenges. J Med Syst 2014;38(9):110

6 Madsen EM, Kaminski MS, Yolton RL. Automated decision making: the role of expert computer systems in the future of optometry. J Am Optom Assoc 1993;64(7):479-489

7 Smith AE, Nugent CD, McClean SI. Evaluation of inherent performance of intelligent medical decision support systems: utilising neural networks as an example. Artif Intell Med 2003;27(1):1-27

8 Azimi P, Benzel EC, Shahzadi S, Azhari S, Mohammadi HR. Use of artificial neural networks to predict surgical satisfaction in patients with lumbar spinal canal stenosis: clinical article. J Neurosurg Spine 2014;20(3):300-305 
9 Azimi P, Mohammadi HR, Benzel EC, Shahzadi S, Azhari S, Montazeri A. Artificial neural networks in neurosurgery. J Neurol Neurosurg Psychiatry 2015;86(3):251-256

10 Rughani AI, Dumont TM, Lu Z, et al. Use of an artificial neural network to predict head injury outcome. J Neurosurg 2010; 113(3):585-590

11 Kang DG, Lehman RA Jr, Lenke LG. Challenges in the classification of adolescent idiopathic scoliosis and the utility of artificial neural networks. Spine J 2013;13(11):1534-1537

12 Naudet F, Falissard B, Boussageon R, Healy D. Has evidence-based medicine left quackery behind? Intern Emerg Med 2015;10(5): 631-634

13 Burgers JS. [Criticism of evidence-based medicine: from reductionism to realism in the application of guidelines]. Ned Tijdschr Geneeskd 2015;159:A8376

14 de Schepper EIT, Overdevest GM, Suri P, et al. Diagnosis of lumbar spinal stenosis: an updated systematic review of the accuracy of diagnostic tests. Spine 2013;38(8):E469-E481

15 Suri P, Rainville J, Kalichman L, Katz JN. Does this older adult with lower extremity pain have the clinical syndrome of lumbar spinal stenosis? JAMA 2010;304(23):2628-2636

16 Yamashita K, Aono H, Yamasaki R. Clinical classification of patients with lumbar spinal stenosis based on their leg pain syndrome: its correlation with 2-year surgical outcome. Spine 2007;32(9):980-985

17 Fardon DF, Milette PC; Combined Task Forces of the North American Spine Society, American Society of Spine Radiology, and American Society of Neuroradiology. Nomenclature and classification of lumbar disc pathology. Recommendations of the Combined task Forces of the North American Spine Society, American Society of Spine Radiology, and American Society of Neuroradiology. Spine 2001;26(5):E93-E113

18 Stucki G, Daltroy L, Liang MH, Lipson SJ, Fossel AH, Katz JN. Measurement properties of a self-administered outcome measure in lumbar spinal stenosis. Spine 1996;21(7):796-803

19 Pratt RK, Fairbank JCT, Virr A. The reliability of the Shuttle Walking Test, the Swiss Spinal Stenosis Questionnaire, the Oxford Spinal Stenosis Score, and the Oswestry Disability Index in the assessment of patients with lumbar spinal stenosis. Spine 2002; 27(1):84-91

20 Overdevest GM, Luijsterburg PA, Brand R, et al. Design of the Verbiest trial: cost-effectiveness of surgery versus prolonged conservative treatment in patients with lumbar stenosis. BMC Musculoskelet Disord 2011;12(1):57

21 Zucherman JF, Hsu KY, Hartjen CA, et al. A prospective randomized multi-center study for the treatment of lumbar spinal stenosis with the X STOP interspinous implant: 1-year results. Eur Spine J 2004;13(1):22-31

22 Izzo R, Guarnieri G, Guglielmi G, Muto M. Biomechanics of the spine. Part I: spinal stability. Eur J Radiol 2013;82(1):118-126

23 Blumenthal C, Curran J, Benzel EC, et al. Radiographic predictors of delayed instability following decompression without fusion for degenerative grade I lumbar spondylolisthesis. J Neurosurg Spine $2013 ; 18(4): 340-346$

24 Lee Y-P, Sclafani J. Lumbar iatrogenic spinal instability. Semin Spine Surg 2013;25(2):131-137

25 Resnick DK, Choudhri TF, Dailey AT, et al; American Association of Neurological Surgeons/Congress of Neurological Surgeons. Guidelines for the performance of fusion procedures for degenerative disease of the lumbar spine. Part 9: fusion in patients with stenosis and spondylolisthesis. J Neurosurg Spine 2005;2(6): 679-685

26 Aghayev K, Gonzalez-Blohm SA, Doulgeris JJ, Lee WE III, Waddell $\mathrm{JK}$, Vrionis FD. Feasibility and biomechanical performance of a novel transdiscal screw system for one level in non-spondylolisthetic lumbar fusion: an in vitro investigation. Spine J 2014;14(4): 705-713
27 Malmivaara A, Slätis P, Heliövaara M, et al; Finnish Lumbar Spinal Research Group. Surgical or nonoperative treatment for lumbar spinal stenosis? A randomized controlled trial. Spine 2007;32(1): $1-8$

28 Yang C, Zheng Z, Liu H, Wang J, Kim YJ, Cho S. Posterior vertebral column resection in spinal deformity: a systematic review. Eur Spine J 2015

29 Dincer F. "Lateral recess syndrome and computed tomography," vol. 2, pp. 30-35, 1991

30 Ciric I, Mikhael MA, Tarkington JA, Vick NA. The lateral recess syndrome. A variant of spinal stenosis. J Neurosurg 1980;53(4): 433-443

31 Lee S, Lee JW, Yeom JS, et al. A practical MRI grading system for lumbar foraminal stenosis. AJR Am J Roentgenol 2010;194(4): 1095-1098

32 Barz T, Melloh M, Staub LP, et al. Nerve root sedimentation sign: evaluation of a new radiological sign in lumbar spinal stenosis. Spine 2010;35(8):892-897

33 Rawall S, Mohan K. “References 1.,"Spine 2010;35(24):1359-1361

34 Khanna AJ. Commentary: the sedimentation sign: correlation with operative level in patients undergoing lumbar decompression for spinal stenosis. Spine J 2013;13(8):843-844

35 Fazal A, Yoo A, Bendo JA. Does the presence of the nerve root sedimentation sign on MRI correlate with the operative level in patients undergoing posterior lumbar decompression for lumbar stenosis? Spine J 2013;13(8):837-842

36 Moses RA, Zhao W, Staub LP, Melloh M, Barz T, Lurie JD. Is the sedimentation sign associated with spinal stenosis surgical treatment effect in SPORT? Spine 2015;40(3):129-136

37 Lee GY, Lee JW, Choi HS, Oh KJ, Kang HS. A new grading system of lumbar central canal stenosis on MRI: an easy and reliable method. Skeletal Radiol 2011;40(8):1033-1039

38 Park H-J, Kim SS, Lee Y-J, et al. Clinical correlation of a new practical MRI method for assessing central lumbar spinal stenosis. Br J Radiol 2013;86(1025):20120180

39 Whitman JM, Flynn TW, Childs JD, et al. A comparison between two physical therapy treatment programs for patients with lumbar spinal stenosis: a randomized clinical trial. Spine 2006; 31(22):2541-2549

40 Whitman JM, Flynn TW, Fritz JM. Nonsurgical management of patients with lumbar spinal stenosis: a literature review and a case series of three patients managed with physical therapy. Phys Med Rehabil Clin N Am 2003;14(1):77-101, vi-vii

41 Maitland G. "Peripheral Manipulation," OXFORD ButterworthHeinemann, 1991

42 Greenman P. Priciples of Manual Medicine. Philadelphia1996

43 Kreiner DS, Shaffer WO, Baisden JL, et al; North American Spine Society. An evidence-based clinical guideline for the diagnosis and treatment of degenerative lumbar spinal stenosis (update). Spine J 2013;13(7):734-743

44 Cohen SP, Bicket MC, Jamison D, Wilkinson I, Rathmell JP. Epidural steroids: a comprehensive, evidence-based review. Reg Anesth Pain Med 2013;38(3):175-200

45 Ghai B, Vadaje KS, Wig J, Dhillon MS. Lateral parasagittal versus midline interlaminar lumbar epidural steroid injection for management of low back pain with lumbosacral radicular pain: a double-blind, randomized study. Anesth Analg 2013;117(1): 219-227

46 Barbagallo GMV, Olindo G, Corbino L, Albanese V. Analysis of complications in patients treated with the X-Stop Interspinous Process Decompression System: proposal for a novel anatomic scoring system for patient selection and review of the literature. Neurosurgery 2009;65(1):111-119, discussion 119-120

47 Burnett MG, Stein SC, Bartels RH. Cost-effectiveness of current treatment strategies for lumbar spinal stenosis: nonsurgical care, laminectomy, and X-STOP. J Neurosurg Spine 2010;13(1): $39-46$ 
48 Bowers C, Amini A, Dailey AT, Schmidt MH. Dynamic interspinous process stabilization: review of complications associated with the X-Stop device. Neurosurg Focus 2010;28(6):E8

49 Kabir SMR, Gupta SR, Casey ATH. Lumbar interspinous spacers: a systematic review of clinical and biomechanical evidence. Spine 2010;35(25):E1499-E1506

50 Kovacs FM, Urrútia G, Alarcón JD. Surgery versus conservative treatment for symptomatic lumbar spinal stenosis: a systematic review of randomized controlled trials. Spine 2011;36(20): E1335-E1351

51 Mikami Y, Nagae M, Ikeda T, Tonomura H, Fujiwara H, Kubo T. Tubular surgery with the assistance of endoscopic surgery via midline approach for lumbar spinal canal stenosis: a technical note. Eur Spine J 2013;22(9):2105-2112

52 Wong AP, Smith ZA, Lall RR, Bresnahan LE, Fessler RG. The microendoscopic decompression of lumbar stenosis: a review of the current literature and clinical results. Minim Invasive Surg 2012;2012:325095

53 Yagi M, Okada E, Ninomiya K, Kihara M. Postoperative outcome after modified unilateral-approach microendoscopic midline decompression for degenerative spinal stenosis. J Neurosurg Spine 2009;10(4):293-299

54 Schulitz KP. [Risk of instability following decompression surgery for lumbar spinal stenosis]. Z Orthop Ihre Grenzgeb 1995;133(3): 236-241 Jan

55 Fox MW, Onofrio BM, Onofrio BM, Hanssen AD. Clinical outcomes and radiological instability following decompressive lumbar laminectomy for degenerative spinal stenosis: a comparison of patients undergoing concomitant arthrodesis versus decompression alone. J Neurosurg 1996;85(5):793-802

56 Kato Y, Panjabi MM, Nibu K. Biomechanical study of lumbar spinal stability after osteoplastic laminectomy. J Spinal Disord 1998; 11(2):146-150

57 Bisschop A, Mullender MG, Kingma I, et al. The impact of bone mineral density and disc degeneration on shear strength and stiffness of the lumbar spine following laminectomy. Eur Spine J 2012;21(3):530-536

58 Rosen C, Rothman S, Zigler J, Capen D. Lumbar facet fracture as a possible source of pain after lumbar laminectomy. Spine 1991;16 (6, Suppl)S234-S238

59 Adams MA, Hutton WC. The mechanical function of the lumbar apophyseal joints. Spine 1983;8(3):327-330

60 Grobler LJ, Robertson PA, Novotny JE, Ahern JW. Decompression for degenerative spondylolisthesis and spinal stenosis at L4-5. The effects on facet joint morphology. Spine 1993;18(11):1475-1482

61 Robertson PA, Grobler LJ, Novotny JE, Katz JN. Postoperative spondylolisthesis at L4-5. The role of facet joint morphology. Spine 1993;18(11):1483-1490

62 Posner I, White AA III, Edwards WT, Hayes WC. A biomechanical analysis of the clinical stability of the lumbar and lumbosacral spine. Spine 1982;7(4):374-389

63 Ghogawala Z, Benzel EC, Amin-Hanjani S, et al. Prospective outcomes evaluation after decompression with or without instrumented fusion for lumbar stenosis and degenerative Grade I spondylolisthesis. J Neurosurg Spine 2004;1(3):267-272

64 Resnick DK, Choudhri TF, Dailey AT, et al; American Association of Neurological Surgeons/Congress of Neurological Surgeons. Guidelines for the performance of fusion procedures for degenerative disease of the lumbar spine. Part 10: fusion following decompression in patients with stenosis without spondylolisthesis. J Neurosurg Spine 2005;2(6):686-691

65 Watters WC III, Baisden J, Gilbert TJ, et al; North American Spine Society. Degenerative lumbar spinal stenosis: an evidence-based clinical guideline for the diagnosis and treatment of degenerative lumbar spinal stenosis. Spine J 2008;8(2):305-310
66 Eck JC, Sharan A, Ghogawala Z, et al. Guideline update for the performance of fusion procedures for degenerative disease of the lumbar spine. Part 7: lumbar fusion for intractable low-back pain without stenosis or spondylolisthesis. J Neurosurg Spine 2014; 21(1):42-47

67 Herkowitz HN, Kurz LT. Degenerative lumbar spondylolisthesis with spinal stenosis. A prospective study comparing decompression with decompression and intertransverse process arthrodesis. J Bone Joint Surg Am 1991;73(6):802-808

68 Burgstaller JM, Porchet F, Steurer J, Wertli MM. Arguments for the choice of surgical treatments in patients with lumbar spinal stenosis - a systematic appraisal of randomized controlled trials. BMC Musculoskelet Disord 2015;16(1):96

69 Benditz A, Grifka J, Matussek J. [Lumbar spinal stenosis. From diagnosis to correct therapy]. Z Rheumatol 2015;74(3):215-224, quiz 225

70 Machado GC, Ferreira PH, Harris IA, et al. Effectiveness of surgery for lumbar spinal stenosis: a systematic review and meta-analysis. PLoS ONE 2015;10(3):e0122800

71 Colaiacomo MC, Tortora A, Di Biasi C, Polettini E, Casciani E, Gualdi GF. [Intervertebral instability]. Clin Ter 2009;160(6):e75-e82

72 Liu N, Wood KB, Schwab JH, et al. Utility of Flexion-Extension Radiographs in Lumbar Spondylolisthesis: A Prospective Study. Spine 2015;40(16):E929-E935

73 Mikhael MA, Ciric I, Tarkington JA, Vick NA. Neuroradiological evaluation of lateral recess syndrome. Radiology 1981;140(1): 97-107

74 Ciric I, Mikhael MA. Lumbar spinal-lateral recess stenosis. Neurol Clin 1985;3(2):417-423

75 Ohba T, Ebata S, Fujita K, Sato H, Devin CJ, Haro H. Characterization of symptomatic lumbar foraminal stenosis by conventional imaging. Eur Spine J 2015;24(10):2269-2275

76 Park H-J, Kim SS, Lee S-Y, et al. Clinical correlation of a new MR imaging method for assessing lumbar foraminal stenosis. AJNR Am J Neuroradiol 2012;33(5):818-822

77 Maus T. Imaging the back pain patient. Phys Med Rehabil Clin N Am 2010;21(4):725-766

78 Houten JK, Errico TJ. Paraplegia after lumbosacral nerve root block: report of three cases. Spine J 2002;2(1):70-75 Jan

79 Lurie JD, Tosteson TD, Tosteson A, et al. Long-term outcomes of lumbar spinal stenosis: eight-year results of the Spine Patient Outcomes Research Trial (SPORT). Spine 2015;40(2):63-76

80 Sengupta DK, Herkowitz HN. Lumbar spinal stenosis. Treatment strategies and indications for surgery. Orthop Clin North Am 2003;34(2):281-295

81 Hansraj KK, Cammisa FP Jr, O'Leary PF, et al. Decompressive surgery for typical lumbar spinal stenosis. Clin Orthop Relat Res $2001 ;(384): 10-17$

82 Hansraj KK, O'Leary PF, Cammisa FP Jr, et al. Decompression, fusion, and instrumentation surgery for complex lumbar spinal stenosis. Clin Orthop Relat Res 2001;(384):18-25

83 Herkowitz HN, Garfin SR, Eismont FJ, Bell GR, Balderston RA. Rothman-Simeone The Spine: Expert Consult. Elsevier Health Sciences; 2011

84 Giannadakis C, Nerland US, Solheim O, et al. Does obesity affect outcomes after decompressive surgery for lumbar spinal stenosis? - A multicenter observational registry-based study. World Neurosurg 2015

85 Lubelski D, Thompson NR, Bansal S, et al. Depression as a predictor of worse quality of life outcomes following nonoperative treatment for lumbar stenosis. J Neurosurg Spine 2015;22(3):267-272

86 Bredow J, Oppermann J, Scheyerer MJ, et al. Lumbar lordosis and sacral slope in lumbar spinal stenosis: standard values and measurement accuracy. Arch Orthop Trauma Surg 2015;135(5): 607-612 